\title{
Cidofovir selectivity is based on the different response of normal and cancer cells to DNA damage
}

Tim De Schutter, Graciela Andrei, Dimitri Topalis, Lieve Naesens and Robert Snoeck*

\begin{abstract}
Background: Cidofovir (CDV) proved efficacious in treatment of human papillomaviruses (HPVs) hyperplasias. Antiproliferative effects of CDV have been associated with apoptosis induction, S-phase accumulation, and increased levels of tumor suppressor proteins. However, the molecular mechanisms for the selectivity and antitumor activity of CDV against HPV-transformed cells remain unexplained.

Methods: We evaluated CDV drug metabolism and incorporation into cellular DNA, in addition to whole genome gene expression profiling by means of microarrays in two $\mathrm{HPV}^{+}$cervical carcinoma cells, HPV immortalized keratinocytes, and normal keratinocytes.

Results: Determination of the metabolism and drug incorporation of CDV into genomic DNA demonstrated a higher rate of drug incorporation in $\mathrm{HPV}^{+}$tumor cells and immortalized keratinocytes compared to normal keratinocytes. Gene expression profiling clearly showed distinct and specific drug effects in the cell types investigated. Although an effect on inflammatory response was seen in all cell types, different pathways were identified in normal keratinocytes compared to immortalized keratinocytes and $\mathrm{HPV}^{+}$tumor cells. Notably, Rho GTPase pathways, LXR/RXR pathways, and acute phase response signaling were exclusively activated in immortalized cells. CDV exposed normal keratinocytes displayed activated cell cycle regulation upon DNA damage signaling to allow DNA repair via homologous recombination, resulting in genomic stability and survival. Although CDV induced cell cycle arrest in HPV immortalized cells, DNA repair was not activated in these cells. In contrast, $\mathrm{HPV}^{+}$cells lacked cell cycle regulation, leading to genomic instability and eventually apoptosis.

Conclusions: Taken together, our data provide novel insights into the mechanism of action of CDV and its selectivity for HPV-transformed cells. The proposed mechanism suggests that this selectivity is based on the inability of $\mathrm{HPV}^{+}$cells to respond to DNA damage, rather than on a direct anti-HPV effect. Since cell cycle control is deregulated by the viral oncoproteins E6 and E7 in $\mathrm{HPV}^{+}$cells, these cells are more susceptible to DNA damage than normal keratinocytes. Our findings underline the therapeutic potential of CDV for HPV-associated malignancies as well as other neoplasias.
\end{abstract}

Keywords: Cervical carcinoma, Cidofovir, DNA damage and repair, Gene expression profiling, Human papillomavirus

\footnotetext{
* Correspondence: robert.snoeck@rega.kuleuven.be

Rega Institute for Medical Research, Laboratory of Virology and

Chemotherapy, KU Leuven, Leuven, Belgium
}

\section{Biomed Central




\section{Background}

Human papillomaviruses (HPVs) are small doublestranded DNA viruses with a strict epithelial tropism. HPVs infect either mucosal or cutaneous surfaces causing a variety of diseases ranging from benign warts (lowrisk types) to malignant neoplasms, including cervical carcinoma and other anogenital cancers (high-risk types) [1]. The virus infects cells in the basal layer of stratified squamous epithelia and viral replication shows both temporal and spatial regulation of viral protein expression. Except for E1 (replicative helicase) and E2 (DNA-binding regulatory protein), HPV completely relies on the cellular DNA synthesis machinery for its genome replication [2]. Development of HPV-induced cancerous lesions is often accompanied by partial integration of the viral genome in the host cell DNA, resulting in conservation and stabilized expression of E6 and E7 oncoproteins [3]. Other parts of the viral genome are usually either deleted or show a disturbed expression [4]. Therefore, cell lines derived from cervical carcinomas do not produce HPV virions and only express the E6 and E7 oncoproteins [5,6].

These two viral oncogenes cooperate in cell transformation and immortalization [7]. The E7 oncoprotein overrides the G1/S checkpoint of the cell cycle through association with the retinoblastoma family of proteins (pRb, p107 and p130). Via induction of their ubiquitinmediated proteolysis, and disruption of their association with the E2f family of transcription factors, E7 activates expression of several S-phase specific genes [8]. E7 also alters cell cycle control through interactions with histone deacetylases, cyclins and cyclin-dependent kinase inhibitors ( $\mathrm{p} 21^{\mathrm{CIP} 1}$ and $\left.\mathrm{p} 27^{\mathrm{KIP} 1}\right)$ that are important regulators of growth arrest during epithelial differentiation [7]. As a result of $\mathrm{pRb}$ degradation, other activities of this tumor suppressor protein, such as DNA repair and maintenance of genomic integrity, are also abrogated. E7 expression causes stabilization and functional impairment of the tumor suppressor protein p53 resulting in stimulation of apoptosis. To counteract this, E6 proteins target p53, leading to ubiquitinylation and proteasomal degradation of p53, preventing cell growth arrest and apoptosis [9]. E6 proteins also activate telomerase expression and regulate the activities of PDZ domain-containing proteins and tumor necrosis factor receptors. Both E6 and E7 induce genomic instability and also target cytokine expression to control cell proliferation and interferon responses [7].

HPV-related malignancies, other than cervical cancer, have increased in the last years because of the higher number of immunocompromised patients. Current treatment modalities for HPV-associated anogenital hyperplasia rely on removal of the lesions and are often mutilating, painful and associated with high recurrence rates. New medical therapies, such as intralesional or topical administration of cidofovir (CDV, Vistide $\left.{ }^{\circledR}\right)$, which maintain the anatomical integrity and sexual function of the patients need to be further investigated. Cidofovir, approved by the FDA for intravenous administration in the therapy of cytomegalovirus retinitis in AIDS patients, has a broad-spectrum anti-DNA virus activity, including HPVs [10]. Its antiviral activity against viruses that encode for their own DNA polymerases (herpes-, pox-, and adenoviruses) is based on a higher affinity of the active diphosphate metabolite (CDVpp) for viral DNA polymerases compared to cellular DNA polymerases [10].

CDV can be used intravenously, intralesionally or topically. Systemic administration requires co-administration of oral probenecid and intravenous hydration to prevent nephrotoxicity. Topical cidofovir is a simple and usually well-tolerated therapy with minimal, if any, side effects (ulcerations at the site of affected mucosa but not on the surrounding normal tissue). These local side effects, when appearing, are self-healing and do not require cessation of treatment. Despite the fact that HPVs do not encode for their own DNA polymerase, off-label use of cidofovir was effective in the treatment of high-risk HPV-associated hyperplasia's including, cervical [11,12], vulvar [13-15], perianal [14], gingival and buccal [16], and hypopharyngeal and esophageal [17] neoplasias.

In vitro, CDV has been shown to exert antiproliferative effects against $\mathrm{HPV}$-positive $\left(\mathrm{HPV}^{+}\right)$cervical carcinoma cells, and to a lower extent against HPV-negative $\left(\mathrm{HPV}^{-}\right)$ immortalized cells [18]. The antiproliferative effect of CDV was ascribed to apoptosis induction, accumulation of cells in S-phase, and induction of p53, pRb and p21 protein expression $[19,20]$. A synergistic effect of CDV and radiation in $\mathrm{HPV}^{+}$cervical carcinoma cells [21] and in head and neck squamous cell carcinoma cells [22] was associated with p53 accumulation. The stromal-derived factor $1(\mathrm{SDF}-1 \alpha)$-stimulated invasiveness of $\mathrm{HPV}^{+}$cells was abrogated by CDV and this anti-metastatic action was mediated by inhibition of E6/E7, CXCR4 and Rho/ ROCK signaling [23]. To explain the selectivity of CDV for HPV-transformed cells, it was suggested that CDV could be differentially metabolized in $\mathrm{HPV} 6^{+}$cells versus human keratinocytes [24]. However, the molecular mechanisms underlying the selectivity of CDV for HPV remain unexplained.

Gene expression profiling has proven successful in identifying the mechanism of action of pharmaceutical agents $[25,26]$. In this study, we evaluated gene expression changes following CDV treatment of different cell types [including, two $\mathrm{HPV}^{+}$cervical carcinoma cell lines (SiHa and HeLa), an HPV ${ }^{-}$immortalized keratinocyte cell line $(\mathrm{HaCaT})$, and primary human keratinocytes (PHKs)] to provide more insights into the mode of action and selectivity of CDV. Furthermore, metabolic studies and drug incorporation into genomic DNA were analyzed in the four cell types. 


\section{Methods}

\section{Antiviral compound}

Cidofovir (CDV), obtained from Gilead Sciences (Foster City, CA, USA), was prepared as $10 \mathrm{mg} / \mathrm{ml}$ solution in PBS. [5- $\left.{ }^{3} \mathrm{H}\right]$-CDV $(1 \mathrm{mCi} / \mathrm{ml}$; specific activity: $26 \mathrm{Ci} / \mathrm{mmol})$ was synthesized by Moravek Biochemicals (Brea, CA, USA), and stored at $-20^{\circ} \mathrm{C}$ in ethanol/water 1:1.

\section{Cell cultures}

The following cell types were used: $\mathrm{HPV}^{+} 6^{+}(\mathrm{SiHa})$ and $\mathrm{HPV}^{+} 8^{+}$(HeLa) cervical carcinoma cell lines, $\mathrm{HPV}^{-}$human immortalized keratinocytes $(\mathrm{HaCaT})$ and primary human keratinocytes (PHKs). SiHa, HeLa and $\mathrm{HaCaT}$ cells were maintained in Dulbecco's modified Eagle's medium (Gibco ${ }^{\circledR}$, Life Technologies ${ }^{\mathrm{Tn}}$, Invitrogen, Belgium) supplemented with $10 \%$ fetal calf serum. PHKs were isolated from neonatal foreskins as described previously [27] and cultured in Keratinocyte-SFM Medium $\left(\right.$ Gibco $^{\circledR}$, Life Technologies $\left.^{\mathrm{Tw}}\right)$.

\section{Total RNA extraction}

Cells pellets containing $10^{6}$ cells were lysed with TRIzol reagent (Invitrogen, Belgium) for 3 minutes at room temperature. Chloroform, $20 \%$ of total volume, was added to the mixture which was subsequently centrifuged $(13,000 \mathrm{rpm})$ at $4^{\circ} \mathrm{C}$ for 15 minutes. The upper aqueous layer containing the RNA was recovered and mixed with an equal volume of $70 \%$ ethanol. The RNA was further purified by RNeasy Mini Kit (Qiagen Benelux, Netherlands) according to manufacturer's instructions. Concentration and purity of RNA was determined with a NanoDrop ND1000 device (Fisher Scientific, Belgium). Integrity of RNA samples was verified by standard denaturing agarose gel electrophoresis. For microarray experiments, RNA quality was also assessed by an Agilent Bioanalyzer system (Agilent, Belgium).

\section{Gene expression profiling by microarrays}

Human Genome U133 Plus 2.0 arrays (Affymetrix, CA, USA) were used to analyze whole genome gene expression in a single hybridization, containing more than 54,000 probe sets and covering approximately 38,500 genes. Array hybridization, scanning and image analyzing were done according to the manufacturer's protocols (Affymetrix GeneChip Expression Assay) at the VIB Nucleomics Core Facility (www.nucleomics.be).

Three different microarray experiments were carried out to evaluate gene expression changes following $50 \mu \mathrm{g} / \mathrm{ml} \mathrm{CDV}$ treatment: experiment ' 1 ' included a wide range of treatment periods (between $2 \mathrm{~h}$ and $120 \mathrm{~h}$ ) of $\mathrm{SiHa}$ cells using one microarray per time point and per condition; experiment ' 2 ' consisted of $\mathrm{SiHa}$ cells treated for $24 \mathrm{~h}, 48 \mathrm{~h}$, and $72 \mathrm{~h}$; experiment ' 3 ' comprised HeLa, $\mathrm{HaCaT}$, and PHK exposed to CDV for $72 \mathrm{~h}$. In the second and third experiments, gene expression profiling was explored by triplicate testing.

\section{Analysis of microarray data}

Raw data were corrected for background signal using the RMA (Robust Multi-array analysis) algorithm (affy_1.22.0 package of BioConductor) that normalizes the data so that different arrays can be compared to each other and summarizes the data into expression values [28]. The detection (Present/Absent) call generated by the Affymetrix microarray suite version 5 software (MAS 5.0) was used to remove probe sets that were not reliable detected in any of the microarrays before further analysis.

Differentially expressed (DE) probe sets between CDV treated and untreated cells were determined using a moderated $t$-statistic test [LIMMA (linear models for microarray data), BioConductor]. The Benjamini Hochberg correction for multiple testing $[\mathrm{p}<0.05$, false discovery rate $(F D R)=0.05]$ was performed [29]. Probe sets were considered significantly $\mathrm{DE}$ if the absolute fold change (FC) was $>2$ and the $P$-value was $<0.05$ (LIMMA) after applying the Benjamini-Hochberg correction. The resulting list of relative gene expression levels for a given condition was designated as a data set (Additional file 1: Figure S1).

\section{Microarray data accession number}

The entire set of microarray data is deposited in the Gene Expression Omnibus (GEO, http://www.ncbi.nlm. nih.gov/projects/geo) according to MIAME standards under accession numbers GSE26748 (SiHa data) and GSE39293 (HeLa, HaCaT, and PHKs data), respectively:

$$
\begin{aligned}
& \text { http://www.ncbi.nlm.nih.gov/geo/query/acc.cgi? } \\
& \text { token=lpivfquymowyazo\&acc=GSE26748 } \\
& \text { http://www.ncbi.nlm.nih.gov/geo/query/acc.cgi? } \\
& \text { token=lbqtpommkiccudo\&acc=GSE39293 }
\end{aligned}
$$

\section{Bioinformatics analysis of differentially expressed (DE) genes}

Ingenuity Pathways Analysis (IPA, Ingenuity ${ }^{\circledR}$ Systems, Redwood City, CA, USA; http://www.ingenuity.com) version 9 was used to perform functional, transcription factor, and canonical pathway analysis. The IPA application reveals relevant pathways and biological functions by comparing the number of genes that participate in a given function or pathway, relative to the total number of occurrences of those genes in all the pathways stored in the IPKB (Ingenuity Pathway Knowledge Base).

Data sets with the corresponding FC and $P$-value were uploaded into the IPA software. Stringent criteria, equivalent to those described for the selection of DE probes, were applied to identify DE genes. When genes were represented 
by 2 or more probe sets on the arrays, only the maximum FC was used. Uncharacterized probe sets were not included in the analysis. Networks were built by determining all interactions among genes categorized with the functional analysis.

\section{RT-PCR analysis}

To validate the microarray data, expression levels of selected genes were determined by real-time RT-PCR using the TaqMan ${ }^{\circledR}$ Fast Universal PCR Master Mix and TaqMan ${ }^{\circledR}$ Gene Expression Assays from Applied Biosystems (Foster City, CA, USA). Equal amounts of total RNA isolated from CDV treated and untreated cells were transcribed to cDNA with the First-Strand cDNA Synthesis Kit (GE Heathcare, Little Chalfont, UK) following manufacturer's instructions. RT-PCR was performed on a 7500 Fast Real-Time PCR System (Applied Biosystems, Foster City, CA, USA) according to manufacturer's instructions. Relative expression levels were calculated with the $\Delta \Delta C_{T}$ method, using $\beta$-actin as endogenous control (Applied Biosystems).

The expression of the two HPV16 oncogenes E6 and E7 in SiHa cells was also quantified with RT-PCR. The cDNA's were prepared as described above and RT-PCR was also carried out under the same experimental conditions. The following forward $(\mathrm{F})$ and reverse $(\mathrm{R})$ primers and probes (P) were used: HPV16_E6_F: 5'-AGAACTGCAATGTT TCAGGACC-3', HPV16_E6_R: 5'-TGTATAGTTGTTTG CAGCTCTGTGC-3', HPV16_E6_P: (FAM) 5' -ACAGGA GCGACCCAGA-3', HPV16_E7_F: 5'-GCTCAGAGGAG GAGGATGAAATAGA-3', HPV16_E7_R: 5'-GAGTCAC ACTTGCAACAAAAGGTT-3', HPV16_E7_P: (FAM) 5'TCCGGTTCTGCTTGTCC-3'.

\section{Metabolism study with $\left[5-{ }^{3} \mathrm{H}\right]-\mathrm{CDV}$}

Radioactive $\left[5-{ }^{3} \mathrm{H}\right]$-labeled CDV was used to evaluate the metabolism in the different cell types. Cells $\left(2 \times 10^{6}\right.$ cells per $75 \mathrm{~cm}^{2}$ flask) were incubated with $\left[5-{ }^{3} \mathrm{H}\right]-\mathrm{CDV}$ at a final concentration of $50 \mu \mathrm{g} / \mathrm{ml}$ and $10 \mu \mathrm{Ci}$ per flask. After $72 \mathrm{~h}$ incubation at $37^{\circ} \mathrm{C}$, samples for HPLC analysis were prepared by methanol extraction as described previously [30]. For HPLC analysis, $200 \mu \mathrm{L}$ extract was injected onto an anion-exchange Partisphere SAX column (dimensions: $4.6 \mathrm{~mm} \times 125 \mathrm{~mm}$ ) from Whatman (Maidstone, UK), and separated with two phosphate buffers (A: $5 \mathrm{mM}$ and B: $0.3 \mathrm{M}$ ammonium dihydrogen phosphate; both at $\mathrm{pH} 3.5$ ), and the following gradient (flow: $2 \mathrm{ml} / \mathrm{min}$ ): $100 \% \mathrm{~A}$ (5 $\mathrm{min}$ ); linear gradient to $100 \%$ B (15 min); $100 \%$ B (20 min); linear gradient to $100 \% \mathrm{~A}(5 \mathrm{~min})$ and $100 \% \mathrm{~A}(15 \mathrm{~min})$. One minute fractions of the eluate were collected, mixed with Hisafe 3 cocktail (Perkin Elmer, Waltham, MA) and analyzed for radioactivity in a scintillation counter. The retention times of the different CDV metabolites were: $3 \mathrm{~min}$ for
CDVp-choline; 5 min for CDV; 15 min for CDVp; and 19 min for CDVpp.

To determine incorporation of $\left[5-{ }^{3} \mathrm{H}\right]-\mathrm{CDV}$ into cellular nucleic acid material, the methanol-insoluble pellets were digested in $500 \mu \mathrm{l} 5 \mathrm{M}$ sodium hydroxide during $24 \mathrm{~h}$ incubation at $37^{\circ} \mathrm{C}$. Sodium hydroxide extracts were neutralized with $500 \mu \mathrm{l} 5 \mathrm{M}$ hydrochloride. Nucleic acid samples were transferred to scintillation vials, mixed with Hisafe 3 cocktail (Perkin Elmer, Waltham, MA) and analyzed for total radioactivity in a scintillation counter. All conditions were performed in duplicate.

\section{Results}

\section{Metabolism and incorporation}

Since CDV has been suggested to be preferentially converted to its active diphosphate form (CDVpp) in $\mathrm{HPV}^{+} 6^{+}$cells [24], we investigated the metabolism of $\left[5-{ }^{3} \mathrm{H}\right]-\mathrm{CDV}$ in $\mathrm{HPV}^{+}$cervical carcinoma cells compared to $\mathrm{HPV}^{-}$immortalized keratinocytes and normal keratinocytes. Following $72 \mathrm{~h}$ incubation with the compound, CDV-phosphocholine (which is considered the intracellular depot form of CDV) appeared to be the most abundant metabolite while the monophosphate form (CDVp) was the least abundant one in all four cell types. No significant differences in the levels of the active metabolite (CDVpp), CDV-phosphocholine or $\mathrm{CDV}$ were observed between PHKs and $\mathrm{HPV}^{+}$tumor cells. However, lower CDVp levels were measured in PHKs compared to $\mathrm{HPV}^{+}$cells following $72 \mathrm{~h}$ incubation.

Notably, lower concentrations of CDV and of all metabolites were observed in $\mathrm{HaCaT}$ cells, compared to either $\mathrm{HPV}^{+}$cells or PHKs (Table 1), suggesting that $\mathrm{HaCaT}$ cells have a different uptake and/or efflux of $\mathrm{CDV}$, rather than differences in drug metabolism.

To compare the efficiency of CDV incorporation into genomic DNA in the different cell types, we performed an analysis of the methanol-insoluble pellets obtained from each cell type after incubation with radiolabeled compound for $72 \mathrm{~h}$. Although the levels of intracellular CDV metabolites were not strikingly different in PHKs compared to immortalized keratinocytes and $\mathrm{HPV}^{+}$ tumor cells, evaluation of the methanol-insoluble fractions revealed important differences between the distinct cell types with higher amounts of CDV incorporated in tumor cells compared to normal keratinocytes. Following $72 \mathrm{~h}$ incubation, $2 \mathrm{pmol}$ of $\mathrm{CDV}$ was found in the methanol-insoluble fraction per million cells for PHKs while at least 4-fold (HaCaT), 6-fold (HeLa) and 9-fold ( $\mathrm{SiHa}$ ) higher levels were determined in the immortalized keratinocytes and $\mathrm{HPV}^{+}$tumor cells (Table 1).

These data indicate that CDVpp is more effective in terms of inhibition of cellular DNA synthesis leading to S-phase arrest for malignant cells than for normal cells. The higher incorporation of CDV into cellular DNA 
Table 1 CDV metabolism and incorporation

\begin{tabular}{|c|c|c|c|c|}
\hline & $\mathrm{SiHa}\left[\mathrm{pmol} / 10 \mathrm{e}^{6}\right.$ cells] & HeLa [pmol/10e ${ }^{6}$ cells] & HaCaT $\left[p m o l / 10 e^{6}\right.$ cells] & PHKs [pmol/10e cells] \\
\hline \multicolumn{5}{|l|}{ Methanol-soluble fraction } \\
\hline CDV & $4.55 \pm 0.03$ & $3.20 \pm 0.25$ & $1.76 \pm 0.11$ & $4.62 \pm 0.63$ \\
\hline CDVp & $2.35 \pm 0.13$ & $1.61 \pm 0.04$ & $0.38 \pm 0.08$ & $0.78 \pm 0.14$ \\
\hline CDVpp & $3.50 \pm 0.02$ & $2.93 \pm 0.08$ & $1.56 \pm 0.01$ & $3.56 \pm 0.28$ \\
\hline CDVp-choline & $11.90 \pm 0.37$ & $8.73 \pm 0.24$ & $2.65 \pm 0.12$ & $9.18 \pm 1.34$ \\
\hline Sum of all metabolites & $22.29 \pm 0.23$ & $16.47 \pm 0.06$ & $6.34 \pm 0.08$ & $18.13 \pm 2.39$ \\
\hline \multicolumn{5}{|c|}{ Digestion of methanol- insoluble fraction } \\
\hline Incorporated CDV & $18.39 \pm 0.54$ & $13.17 \pm 1.43$ & $8.62 \pm 1.11$ & $1.96 \pm 0.71$ \\
\hline
\end{tabular}

Cells were incubated for $72 \mathrm{~h}$ with $10 \mu \mathrm{Ci}$ radiolabeled CDV (final concentration of $50 \mu \mathrm{g} / \mathrm{ml}$ ) to asses metabolism and incorporation in cellular DNA. Data are the mean \pm SD of two experiments.

observed in $\mathrm{HPV}^{+}$malignant cells compared to normal cells is in agreement with the selectivity of this compound for tumor cells. To investigate the consequences of this differential incorporation of CDV into cellular DNA, whole human genome gene expression profiling was performed.

\section{Gene expression profiling Kinetic study of gene expression changes}

First, a kinetic study was performed to assess gene expression changes in $\mathrm{SiHa}$ cells incubated in the presence or absence of CDV for different times (from $2 \mathrm{~h}$ to $120 \mathrm{~h}$ ). Considering the minimal changes observed up till $24 \mathrm{~h}$ following CDV addition, a second kinetic was performed that included treatment for $24 \mathrm{~h}, 48 \mathrm{~h}$ and $72 \mathrm{~h}$. After $24 \mathrm{~h}$, only 2 genes (DHRS2 and HIST1H2AC) were downregulated, while no genes were found to be upregulated. Venn diagrams (Figure 1A) were used to classify the total number of genes whose expression change was specific to or common in the comparisons of CDV treatment for $24 \mathrm{~h}, 48 \mathrm{~h}$ and $72 \mathrm{~h}$. The number of differentially expressed (DE) genes increased with the duration of CDV exposure. A total of 27 and 140 genes were DE after, respectively, $48 \mathrm{~h}$ and $72 \mathrm{~h}$ of CDV administration, the majority of the genes being upregulated (17 and 108 genes after $48 \mathrm{~h}$ and $72 \mathrm{~h}$, respectively). Out of the 27 genes that showed an altered expression level following $48 \mathrm{~h}$ of treatment with CDV, 20 showed a similar alteration after $72 \mathrm{~h}$ (Figure 1A).

\section{Comparison of gene expression profiling among different cell types}

Based on the kinetic study and taking into account the overlap between the $48 \mathrm{~h}$ and $72 \mathrm{~h}$ data, the impact of CDV on gene expression in different cell types was evaluated at $72 \mathrm{~h}$ post-administration of the compound. To investigate the selectivity of CDV for $\mathrm{HPV}^{+}$tumor cells and whether the presence of HPV affects the response to $\mathrm{CDV}$, an $\mathrm{HPV} 18^{+}$carcinoma cell line (HeLa), an $\mathrm{HPV}^{-}$ immortalized keratinocyte cell line ( $\mathrm{HaCaT})$, and normal keratinocytes (PHKs) were evaluated in addition to $\mathrm{SiHa}$ cells (Additional file 1: Figure S1).

A comparison of the total number of genes that were found to be DE among the four cell types is depicted with Venn diagrams (Figure 1B). Similarly to SiHa cells, most of the DE genes were upregulated in HeLa, HaCaT and PHKs. The number of genes with deregulated expression was higher in $\mathrm{HPV}^{-}$than in $\mathrm{HPV}^{+}$cell types. The vast majority of DE genes following CDV incubation did not overlap between the different cell types. Only two genes (AOX1 and CLIC3) were upregulated in all four tested cell types. Genes with reduced expression levels common to all four cell types were not detected (Figure 1B).

Different types of analysis (functional, upstream regulator, and pathway analysis) were performed with the four microarray data sets through the use of Ingenuity Pathways Analysis (IPA, Ingenuity ${ }^{\circledR}$ Systems). A comparison of the functional annotations upregulated or downregulated following CDV treatment in the four cell types is shown in Additional file 2: Figure S2 and a complete list with all identified canonical pathways affected by CDV is given in Additional file 3: Table S1. The upstream regulator analysis, a novel approach to transcription factor prediction, was used to predict activation or inhibition of transcription factors to describe gene expression alterations in our data set (Additional file 4: Table S2). In addition, IPA was used to generate networks which are graphical representation of molecular relationships between different genes.

\section{Validation of gene expression changes by RT-PCR}

To validate the microarray data, the expression of selected genes (AOX1, DHRS2, HIST1H2AC, ICAM4, MAP2K6, and $O S M R$ ) was quantified by real time RT-PCR. Genes that were found to be up- or downregulated by CDV in the microarray data were confirmed by RT-PCR assay (Figure 2A) while those that were not DE in the microarray data showed similar results by RT-PCR. Only a 


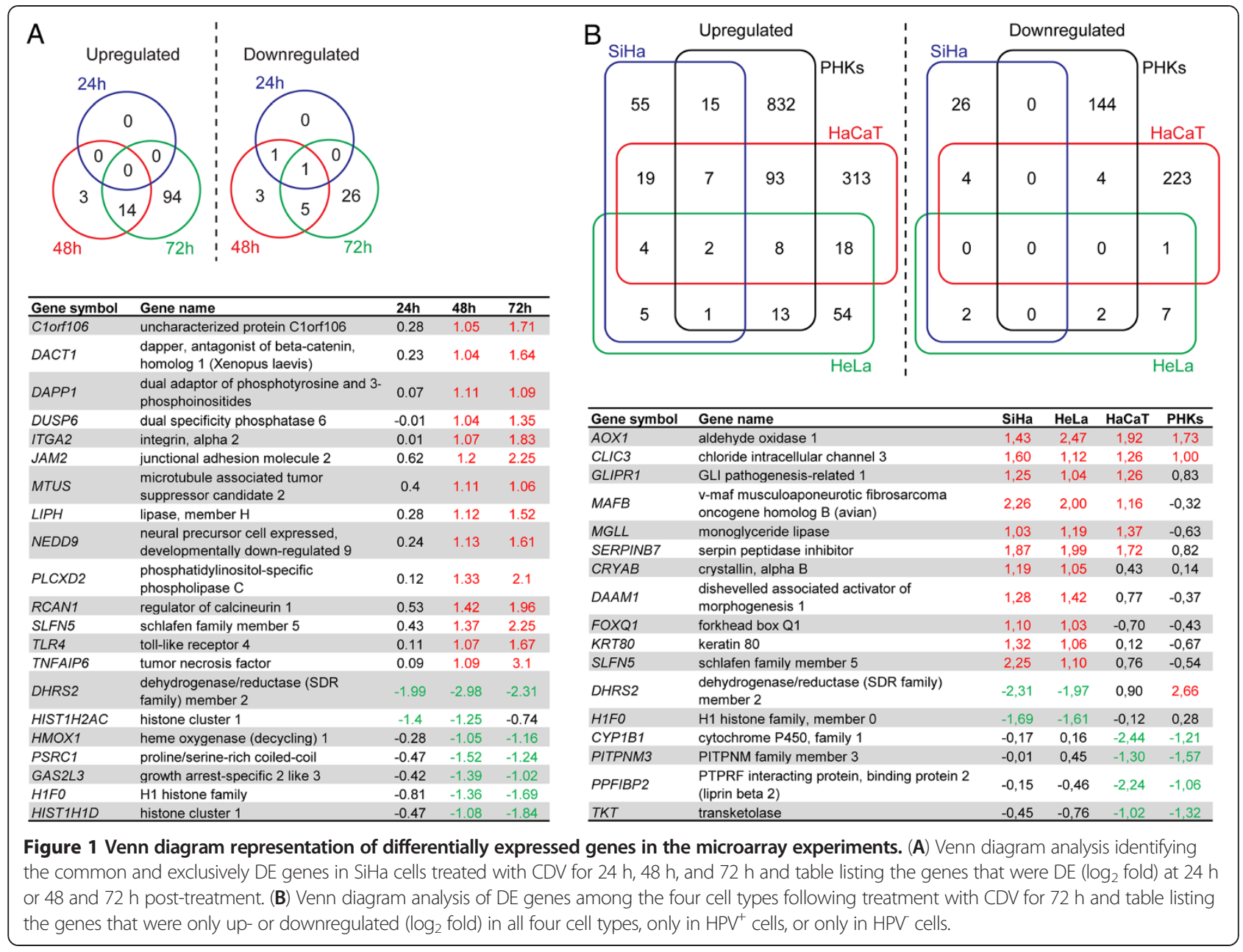

minor difference was observed in the relative expression level of DHRS2 in HaCaT cells. This gene was 1.9-fold upregulated in the microarray data, which was just below the cut-off (2-fold DE), while being 2.9-fold upregulated in the RT-PCR assay.

Considering that HPV abrogates the functions of the p53 and pRb tumor suppressor proteins and that CDV treatment results in increased levels of these two proteins [19], we also evaluated TP53 and RB1 mRNA levels by RT-PCR. Similar to the microarray data, no changes in expression levels of TP53 and RB1 were registered by RT-PCR (Figure 2A). Thus, increased p53 and pRb proteins levels following treatment with CDV reflect posttranscriptional regulation of these genes.

\section{CDV activates the inflammatory response by different mechanisms in immortalized cells and PHKs}

A comparison of the functional annotations affected by CDV in either of the four cell types revealed 'immune response' and 'inflammatory response' to be the only functions upregulated in the different cell types
(Figure 3). However, canonical pathway analysis showed that the effect of CDV on immune response pathways is different for immortalized keratinocytes and $\mathrm{HPV}^{+}$tumor cells compared to normal keratinocytes (Additional file 3: Table S1). Despite the lower number of DE genes in immortalized keratinocytes and $\mathrm{HPV}^{+}$tumor cells than in $\mathrm{PHKs}$, a higher proportion of pathways related to immune response was seen in these cells: $3 / 9$ in $\mathrm{SiHa}, 21 / 53$ in HeLa, 31/57 in HaCaT, compared to 5/35 in PHKs.

Networks were then constructed with DE genes related to inflammatory response (Additional file 5: Figure S3), showing a distinct drug effect on this function in the different cell types. Pathways included in the 'inflammatory response' networks showed that CDV modulated several inflammation-associated signaling pathways in immortalized cells and $\mathrm{HPV}^{+}$tumor cells: 'Acute Phase Response Signaling' in SiHa, HeLa and HaCaT cells; 'Activation of IRF by Cytosolic Pattern Recognition Receptors', 'IL-10 Signaling', 'IL-6 Signaling', 'p38 MAPK Signaling', 'TREM1 Signaling', 'Interferon Signaling' in HeLa and HaCaT cells; 'ILK Signaling', 'Oncostatin M Signaling', and 'Role of 


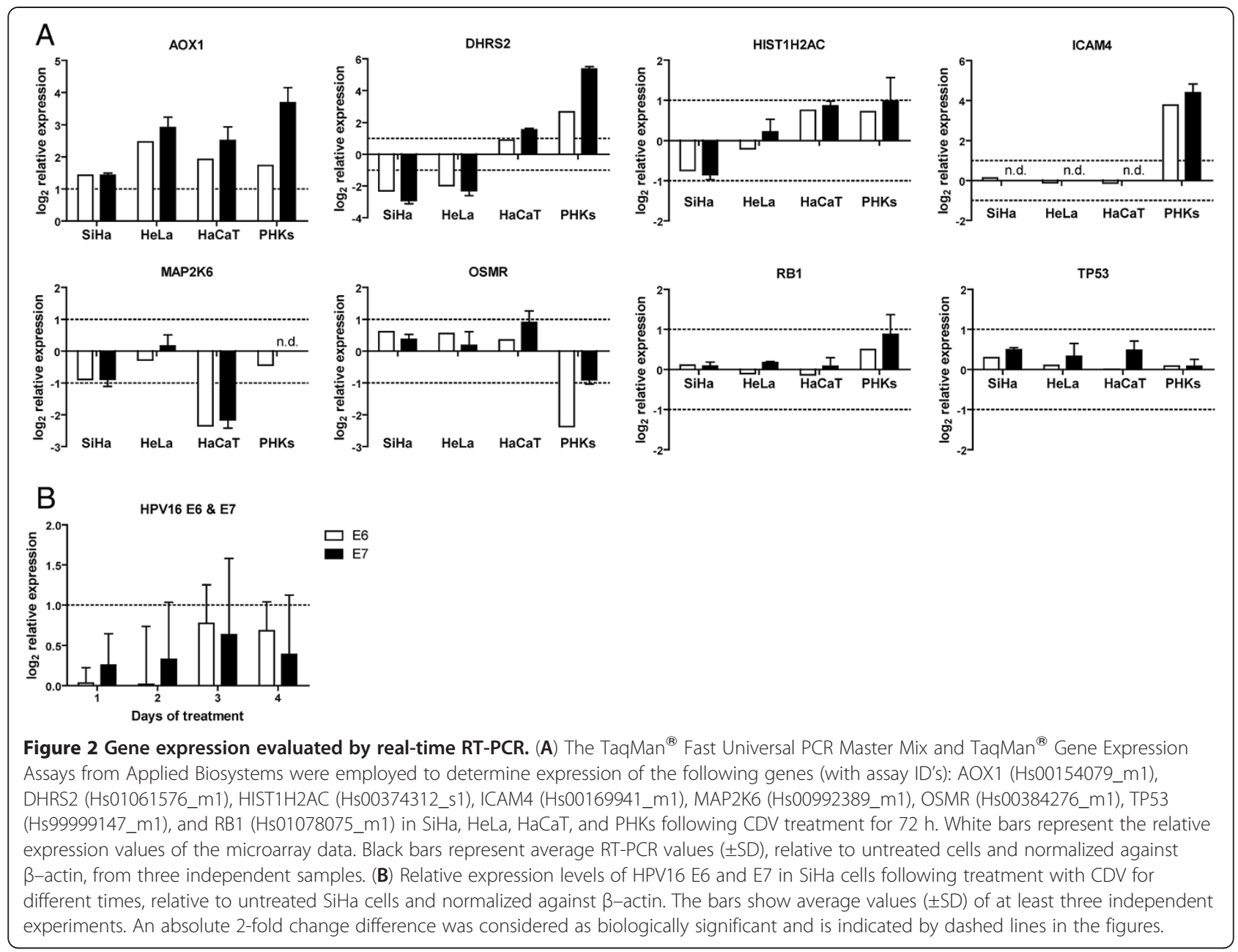

RIG1-like Receptors in Antiviral Innate Immunity' in HeLa cells; 'Toll-like Receptor Signaling' in SiHa cells; and 'HMGB1 Signaling', 'IL-15 Production', 'IL-17 Signaling', 'IL-8 Signaling', 'NF-кB Signaling', and 'OX40 Signaling' in $\mathrm{HaCaT}$ cells (Additional file 5: Figure S3). In contrast, only two pathways ('Interferon Signaling' and 'Role of IL-17A in Psoriasis') related to inflammatory response were recognized in PHKs.

Among the DE genes involved in inflammatory response, solely one gene (AOX1) was found to be upregulated in all four cell types while MGLL was the only gene upregulated in the immortalized keratinocytes and $\mathrm{HPV}^{+}$tumor cells (Additional file 5). Few genes [such as the adhesion molecule NEDD9 ( $\mathrm{SiHa}$ and HeLa) and several genes involved in interferon signaling $(\mathrm{HaCaT})]$ were upregulated both in normal keratinocytes and in one of the immortalized cells.

Increased expression of pro-inflammatory cytokines (IL1, IL6, IL18), genes involved in cytokine-cytokine signaling cascades (IL1R, IL6R, TNFAIP3, TNFAIP6, TNFSF13B, PTX3), cell-cell adhesion (CDH1, ICAM1, FN1, ITGA2), tissue remodeling (VEGF), extracellular matrix $(E C M)$, and proteolysis (SERPINE1) characterized the inflammatory response induced by CDV in immortalized keratinocytes and $\mathrm{HPV}^{+}$tumor cells. Also, regulators of cytokine signaling and NF-kB activation (SPHK1, IRAK2), enzymes involved in the synthesis of prostaglandins (PTGS1), deubiquinating enzymes $(C Y L D)$, and members of the G-protein coupled receptor superfamily (ADRB2) were upregulated in these cells. In PHKs, the inflammatory response was mainly driven by upregulation of genes involved in interferon signaling, including IFIT1, IRF1, OAS1, and STAT1.

Most of the DE genes in the PHKs 'inflammatory response' network were not affected in the other cell types. Moreover, some of the genes in these networks were oppositely affected in PHKs versus immortalized keratinocytes and $\mathrm{HPV}^{+}$tumor cells: extracellular matrix protein tenastatin (TNC) downregulated in PHKs and upregulated in $\mathrm{SiHa}$ and $\mathrm{HaCaT}$ cells; topoisomerase $T O P 2$, lipoxygenase $A L O X 5$, mitogen-activated protein 


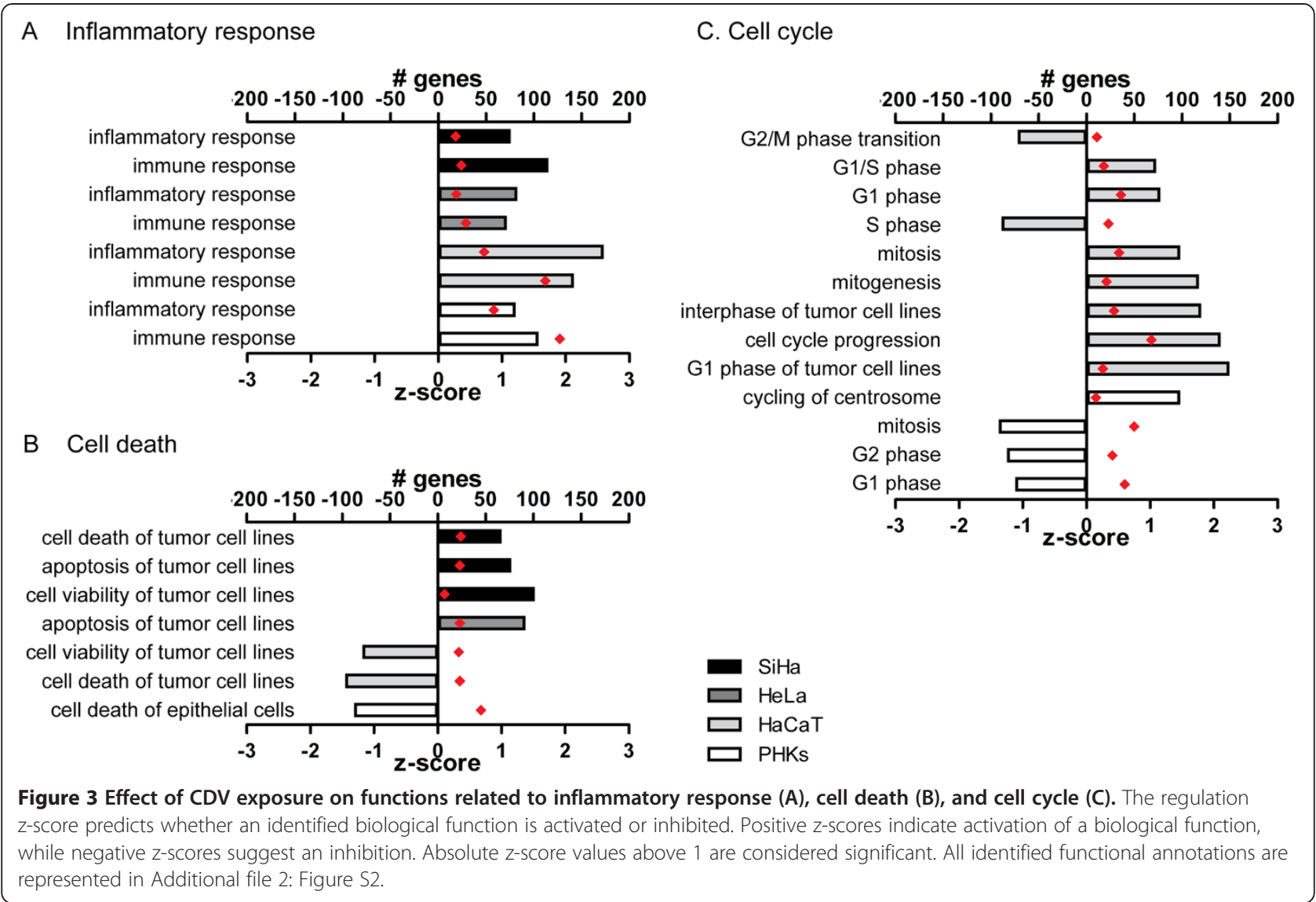

kinase $M A P 3 K 8$, aminopeptidase ERAP1, and PDZ binding kinase $P B K$ upregulated in $P H K s$ and downregulated in $\mathrm{HaCaT}$ cells; transforming growth factor TGFB2 and transcriptional regulator NUPR1 upregulated in $\mathrm{HaCaT}$ and downregulated in PHKs; myosin light chain kinase MYLK upregulated in HeLa cells and downregulated in PHKs.

Retinoid X receptor (RXR) pathways are distinctly affected by CDV in immortalized cells and PHKs

Retinoid X receptors (RXRs) are nuclear receptors which are ligand-regulated transcription factors that modulate development, differentiation, and homeostasis. They recognize target genes by binding to specific DNA recognition sequences, known as hormone response elements. RXRs are important heterodimer partners for many nuclear receptors, including vitamin D3 receptors (VDRs) and liver X receptors (LXRs) [31].

Activation of LXR/RXR pathways following CDV treatment was exclusively observed in the immortalized keratinocytes and $\mathrm{HPV}^{+}$tumor cells (Additional file 3: Table S1) and was associated with increased mRNA levels of the toll-like receptor TLR4, ABC transporters (ABCG1, ABCA1), inflammatory cytokines (IL1A, IL6,
IL18), cytokine receptors (IL1R1, IL1RN, IL1R2, IL36RN), matrix metallopeptidase (MMP9), and/or cyclooxygenase (PTGS2). Activation of LXR/RXR pathways was also linked to downregulation of genes involved in fatty acid biosynthesis such as $S C D$ and of the E3 ubiquitin-protein ligase (MYLIP) in $\mathrm{HaCaT}$ cells. Except for ARG2 that was upregulated in $\mathrm{HaCaT}$ and downregulated in PHKs, these genes were not affected in PHKs.

In contrast to $\mathrm{HPV}^{+}$cells, activation of the VDR/RXR signaling pathway was recorded in $\mathrm{HaCaT}$ and PHKs, yet DE genes implicated in this pathway were rather different between these two cell types. Only increased expression of cystatin CST6 (implicated in tumor suppression) and of the dehydrogenase HSD17B2 (involved in sterol metabolism) were common to both PHKs and $\mathrm{HaCaT}$.

\section{Rho GTPase pathways were affected by CDV exclusively} in immortalized keratinocytes and $\mathrm{HPV}^{+}$tumor cells Pathway analysis showed that changes in Rho GTPase pathways were solely observed in the immortalized cells and $\mathrm{HPV}^{+}$tumor cells: 'RhoGDI Signaling' in both $\mathrm{HPV}^{+}$ cells; 'Rac Signaling' in SiHa cells; 'RhoA Signaling', 'Regulation of Actin-based Motility by Rho', and 'Signaling by Rho Family GTPases' in HeLa cells; and 'Cdc42 
Signaling' in HaCaT (Additional file 3: Table S1). Genes upregulated by CDV that were associated with these pathways encompassed: several members of the major histocompatibility (HLA) complex, different receptors (estrogen receptor ESR1, NK cell-activating receptor $N C R 2$, extracellular matrix receptor $C D 44)$, several regulators of the Rho family of GTPases [E-cadherin ( $C D H 1)$, integrin alpha-2 (ITGA2), myosin (MYL9, MYLK), the ras GTPase-activating-like protein IQGAP2, the insulin-like growth factor 1 IGF1, actins (ACTA2, ACGTG2, ACTC1), the Rho GTPase binding protein $C D C 42 E P 3]$ and a member of the Abelson family of nonreceptor tyrosine protein kinases $A B C L 2$. Only three genes (the Rho GDP dissociation inhibitor $A R H G D I A$, the transcription factor FOS, and the protein kinase PRKCA) involved in Rho GTPase pathways were downregulated by CDV in immortalized cells. Except for MYL9 and MYLK that were oppositely regulated in PHKs versus immortalized keratinocytes and $\mathrm{HPV}^{+}$tumor cells, none of these genes was DE in normal keratinocytes after CDV exposure. Interestingly, another Rho GDP dissociation inhibitor $A R H G D I B$ was upregulated in PHKs.

\section{Specific gene expression signatures in $\mathrm{HPV}^{+}$tumor cells and immortalized keratinocytes treated with CDV}

Four genes (GLIPR1, MAFB, MGLL, and SERPINB7) were exclusively induced by $C D V$ in all three immortalized cells (Figure 1B). These genes are involved in cell death (GLIPR1 and MAFB), growth of cells (SERPINB7), differentiation $(M A F B)$, and migration (MAFB and $M G L L)$. Furthermore, $M G L L$ was associated with lipid metabolism which plays a critical role in malignancy of cancer cells [32] and indeed, lipid metabolism was affected by CDV in HeLa and $\mathrm{HaCaT}$ cells (Additional file 2: Figure S2).

Functions related to cancer encompassed the largest number of genes (and most significant $P$-values) in all tested cell types. While a significant $\mathrm{z}$-score for functions related to cancer was calculated in the immortalized cells, functional annotations associated with malignant transformation had a non-significant z-score in PHKs (Additional file 2: Figure S2).

Based on DE of target genes following exposure to CDV, activation or inhibition of transcription factors was predicted by means of upstream regulator analysis with IPA (Additional file 4: Table S2). In SiHa cells, solely MYCN activities showed a significant negative $\mathrm{z}$-score and thus predictive of a decreased activity. Based on z-score values, decreased activities of MYCN were determined in all three immortalized cells although a non-significant $P$-value was calculated for $\mathrm{SiHa}$ and $\mathrm{HaCaT}$ cells. Activities of the MYC transcription factor, another member of the MYC family of transcription factors, were predicted to be inhibited in HeLa and HaCaT cells.

\section{Selectivity of CDV for $\mathrm{HPV}^{+}$tumor cells: induction of apoptosis}

The functional annotation 'apoptosis of tumor cell lines' was activated following CDV treatment in $\mathrm{HPV}^{+}$cells (Figure 3). Specific sets of genes linked to cell death of tumor cells appeared to be altered following CDV treatment (Figure 4A-B). Most of these genes were only affected in $\mathrm{SiHa}$ and/or HeLa cells but not (or reversely) affected in PHKs. Among others, downregulation of $M D M 4$ and ARHGDIA and upregulation of $B I K$ and $C Y L D$ in SiHa cells, and upregulation of $D K K 3, M Y L K$, $P L A U$, and TIMP3 in HeLa cells, were associated with induction of cell death. Upregulation of CRYAB in $\mathrm{HPV}^{+}$ cells was linked to both decreased apoptosis and decreased growth of cells, reflecting the diverse effects described for this gene. The association of DE genes with pathways related to apoptosis signaling was highlighted in the cell death networks built for the malignant cells (Figure 4A-B).

In contrast to $\mathrm{HPV}^{+}$cells, $\mathrm{HaCaT}$ showed decreased 'cell death of tumor cells' and 'cell viability of tumor cells lines' following CDV treatment (Figure 3). Pathways affected by CDV identified in the cell death network built for $\mathrm{HaCaT}$ were different from those found in $\mathrm{HPV}^{+}$ cells and included 'p53 Signaling', 'Aryl Hydrocarbon Receptor Signaling', 'HGF Signaling', and 'JAK/STAT Signaling' (Figure 4C).

\section{CDV affects cell cycle regulation differently in} immortalized keratinocytes versus normal keratinocytes

Functional analysis suggested distinct effects of CDV on cell cycle in PHKs and $\mathrm{HaCaT}$, while no functional annotations associated with cell cycle were identified in $\mathrm{HPV}^{+}$ cells (Figure 3). Similarly, pathways related to cell cycle control were mainly identified in $\mathrm{HaCaT}$ and PHKs (Additional file 3: Table S1). Although the activities of the transcription factor p53 were activated in HeLa and HaCaT (Additional file 4: Table S2), the 'p53 Signaling' pathway was affected in $\mathrm{HaCaT}$ and normal keratinocytes but not in $\mathrm{HPV}^{+}$cells, with TP63 (member of the p53 transcription factor family) downregulated in PHKs and upregulated in $\mathrm{HaCaT}$.

Distinct sets of genes involved in pathways related to 'cell cycle' and 'DNA replication, recombination, and repair' were altered in $\mathrm{HaCaT}$ and PHKs. Several cyclins and cyclin-dependent kinases (CDKs) that play a key role in cell cycle control were differentially modulated by CDV in HaCaT and PHKs: CCNA2 and CCNB1 were downregulated in $\mathrm{HaCaT}$ and upregulated in $\mathrm{PHKs}$; $C D K 1, C D K 6$, and CCNE2 were upregulated in PHKs, but not in $\mathrm{HaCaT}$.

Prediction of transcription factor activities also showed significant differences between PHKs and $\mathrm{HaCaT}$ (Additional file 4: Table S2). Notably, SMARCB1 (that 


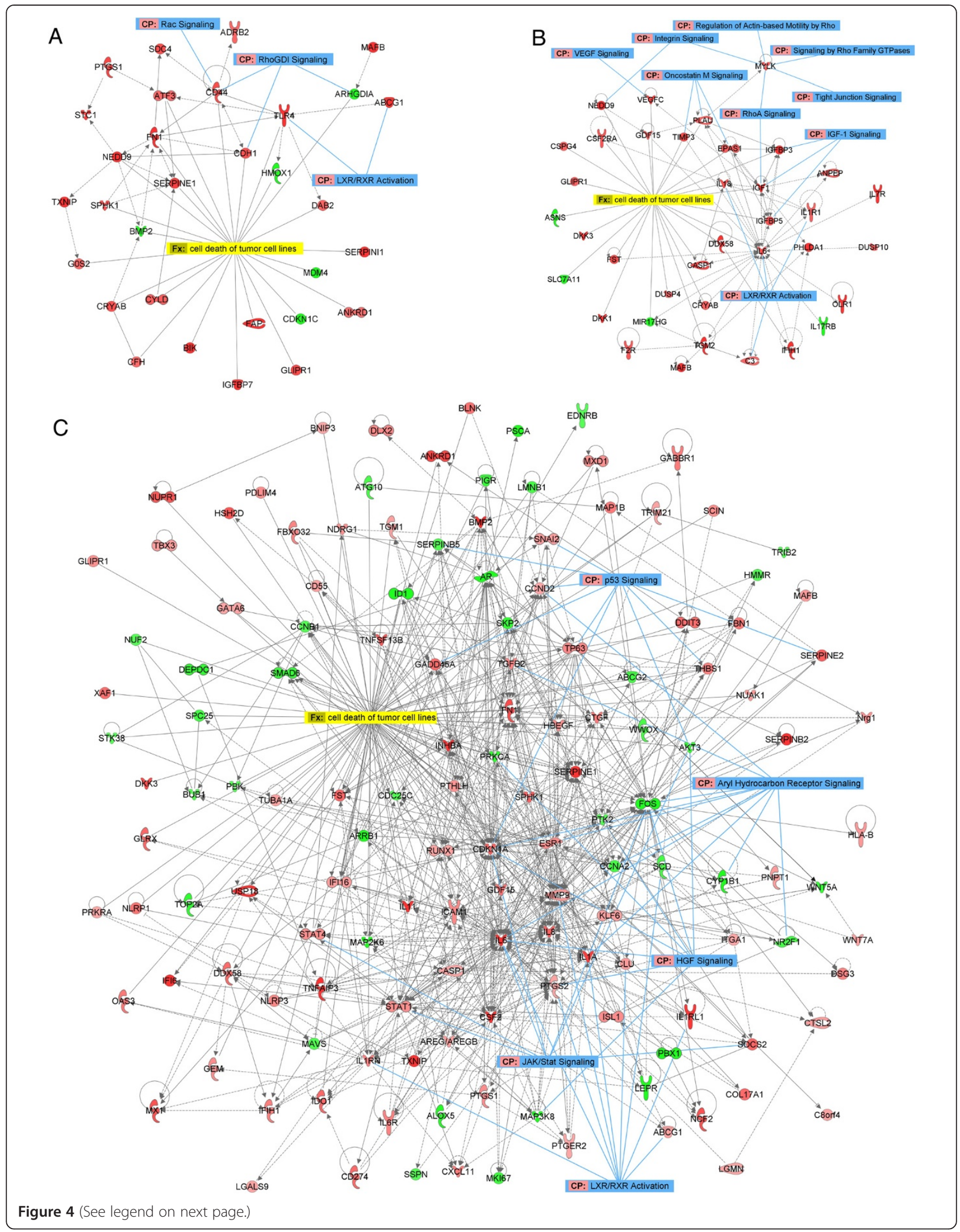



using genes DE and involved in 'cell death of tumor cell lines' following CDV treatment of (A) SiHa, (B) HeLa, or (C) HaCaT cells. A network is a graphical representation of the molecular relationships between molecules (nodes). The biological relationship between two nodes is represented as an edge connecting two nodes. All edges are supported by information from the literature stored in the Ingenuity Pathways Knowledge Base. The intensity of the node color indicates the degree of up-regulation (red) or down-regulation (green) following CDV treatment. Canonical pathways identified by IPA in the networks are shown in blue. Except for DAB2, SERPINI1, CFH, and NEDD9 in SiHa; NEDD9, DKK1, DDX58, CDF15, DUSP4, CASP1, IGFBP3, F2R, SLC7A11, and ASNS in HeLa; XAF1, TRIM21, USP18, TNFSF13B, STAT4, GLRX, DDX58, GEM, TBX3, BMP2, TUBA1A, STAT1, CTGF, IL8, GDF15, CYP1B1, PTHLH, THBS1, CASP1, IL11, CLU, and CTSL2 in HaCaT; no changes in expression for genes included in the cell death networks built for the immortalized cells were observed in PHKs. Notably, BMP2 (SiHa); MYLK (HeLa); and NUPR1, NUF2, SPC25, BUB1, TOP2A, DEPC1, CCNB1, PBK, TGFB2, TP63, ABCG2, CCNA2, MKI67, SSPN, NRG1, MAP3K8, and ALOX5 (HaCaT) were inversely regulated in PHKs.

relieves repressive chromatin structures) predicted functions were activated in $\mathrm{HaCaT}$, but inhibited in PHKs. TBX2 [that suppresses the expression of CDK inhibitors such as p15 and p21 and destabilizes p53 by suppressing expression of ARF (an inhibitor of MDM2)] predicted functions were inhibited in $\mathrm{HaCaT}$ but activated in PHKs. Other transcription factors appeared to be either activated or inhibited exclusively in $\mathrm{HaCaT}$ or PHKs, but not in both. Thus, the activities of the tumor suppressor SMARC4A (that mediates ATP-dependent chromatin remodeling processes) and of the histone demethylase KDM5B (that associates with and contributes to the repression of E2f-target genes during senescence) were exclusively activated in $\mathrm{HaCaT}$ cells. Furthermore, by inhibiting CDKs, the tumor suppressor p16 (CDKN2A), whose predicted activities were upregulated in $\mathrm{HaCaT}$ cells, triggers the G1-S checkpoint that is often considered to be crucial for inducing a senescence-like growth arrest. In line with growth arrest in $\mathrm{HaCaT}$ cells, are the decreased predicted activities of the E2f transcription factor (regulator of expression of genes such as cyclins, CDKs, components of the pre-replication, and DNA synthesis genes) and the increased predicted activities of the chromatin associated protein HMGB1 (that facilitates p53 phosphorylation after to genotoxic stress) and of $\mathrm{NF}_{\mathrm{K} B}$ (a pleiotropic transcription factor that can be activated by DNA damage).

The occurrence of cell cycle arrest in $\mathrm{HaCaT}$ was further evidenced by upregulation of CDKN1A (p21) and downregulation of CCNA2, CCNB1, TOPA2, SKP2, $H D A C 8$, and $P P M 1 L$, in contrast to PHKs (Additional file 6: Table S3).

\section{Specific gene expression signatures in PHKs exposed to CDV Activation of metabolic pathways}

Whereas immortalized keratinocytes and $\mathrm{HPV}^{+}$tumor cells were found to have more alterations in immune response pathways compared to the PHKs, seventeen different pathways (out of 35) linked to metabolism were seen in PHKs versus only one (SiHa), two $(\mathrm{HaCaT})$ and three (HeLa) in CDV-treated immortalized cells (Additional file 3: Table S1).

\section{DNA damage response and survival of epithelial cells}

Pathways related to DNA repair ('ATM Signaling' and 'DNA Double-Strand Break Repair by Homologous Recombination') were exclusively identified in PHKs, suggesting activation of DNA repair mechanisms following CDV-induced DNA damage. Several cell division cycle $(\mathrm{CDC})$ homologs (such as $C D C 2, C D C 6$, $C D C 7, C D C 23$, and $C D C 25 A$ ), that play important roles in cell cycle transition and DNA replication (Additional file 6: Table S3), were exclusively upregulated in PHKs. In contrast, $C D C 25 C$ was found downregulated in $\mathrm{HaCaT}$. Expression of genes encoding for proteins involved in DNA repair and checkpoint control (including ANAPC7, BRCA1, CDT1, CKS2, EME1, GEN1, KAT2B, KIF11, MDM2, NBN, ORC6, PCNA, RAD51, and RFC3) were solely upregulated in PHKs (Additional file 6: Table S3).

Importantly, functional analysis revealed a decrease (z-score $<1$ ) of 'cell death of epithelial cells' following CDV treatment of PHKs, in contrast to increased ( $\mathrm{z}$-score $>1)$ 'cell death of tumor cell lines' in SiHa and HeLa (Figure 3). The upregulation of anti-apoptotic genes (such as BIRC5) in PHKs suggested a successful response to DNA damage.

\section{Discussion}

In this study, the basis for selectivity of CDV for $\mathrm{HPV}^{+}$ tumor cells could be demonstrated based on analysis of drug incorporation into genomic DNA as well as gene expression profiling in $\mathrm{HPV}^{+}$tumor cells, $\mathrm{HPV}^{-}$immortalized keratinocytes and normal keratinocytes. Bioinformatics analysis of microarray data highlighted distinct responses to CDV exposure in PHKs compared to $\mathrm{HPV}^{+}$ cervical carcinoma cells, on one hand, and to $\mathrm{HPV}^{-}$immortalized keratinocytes, on the other hand.

Our findings indicate that the selectivity of CDV for HPV-transformed cells is based on differences in response to DNA damage, replication rate and CDV incorporation into cellular DNA between immortalized cells and PHKs, rather than a specific effect of the drug on the viral oncogenes. However, the presence of E6 and E7 indirectly contributes to the efficacy and selectivity of CDV, because viral oncoproteins deregulate cell cycle, 
impeding cell cycle checkpoints and DNA repair, thus favoring the antiproliferative effects of CDV.

Gene expression profiling of CDV-treated $\mathrm{HaCaT}$ and PHKs revealed specific signatures that clearly explain a differential outcome in both cell types following drug exposure (Figure 5). Except for CYP1B1 and THBS1, complete different sets of genes in pathways related to 'cell cycle' and 'DNA replication, recombination, and repair' were modulated following $\mathrm{CDV}$ exposure of $\mathrm{HaCaT}$ and PHKs, supporting a differential effect on 'cell cycle' functions in immortalized and normal keratinocytes. Interestingly, mRNA levels of many genes involved in these functions were oppositely regulated by CDV in PHKs and in $\mathrm{HaCaT}$ cells or exclusively affected in one of the cell types. HaCaT cells respond to CDV by attempting cell cycle regulation which fails because of the inability of these cells to repair DNA damage (Figure 5). This is further sustained by CDV triggering of 'p53 Signaling' in $\mathrm{HaCaT}$ and normal keratinocytes but not in cervical cancer cells. Also, the prediction of transcription factor activities points to cell cycle arrest in $\mathrm{HaCaT}$ but not in PHKs.

Specific signatures identified in CDV-treated PHKs point to cell cycle regulation and activation of DNA double-strand breaks (DSBs) repair mechanism ('ATM Signaling' and 'DNA Double-Strand Break Repair by Homologous Recombination'), suggesting that CDV can generate DSBs. Homologous recombination (HR) is a conservative process that tends to restore the original DNA sequence at the site of damage. Expression of genes involved in DNA repair by non-homologous endjoining (NHEJ) was not seen in CDV-treated PHKs. This points to a non-mutagenic CDV effect as NHEJ can be mutagenic because it mediates repair by directly ligating the ends of DSBs together, in contrast to HR that is considered a faithful DNA repair process [33]. Since CDV

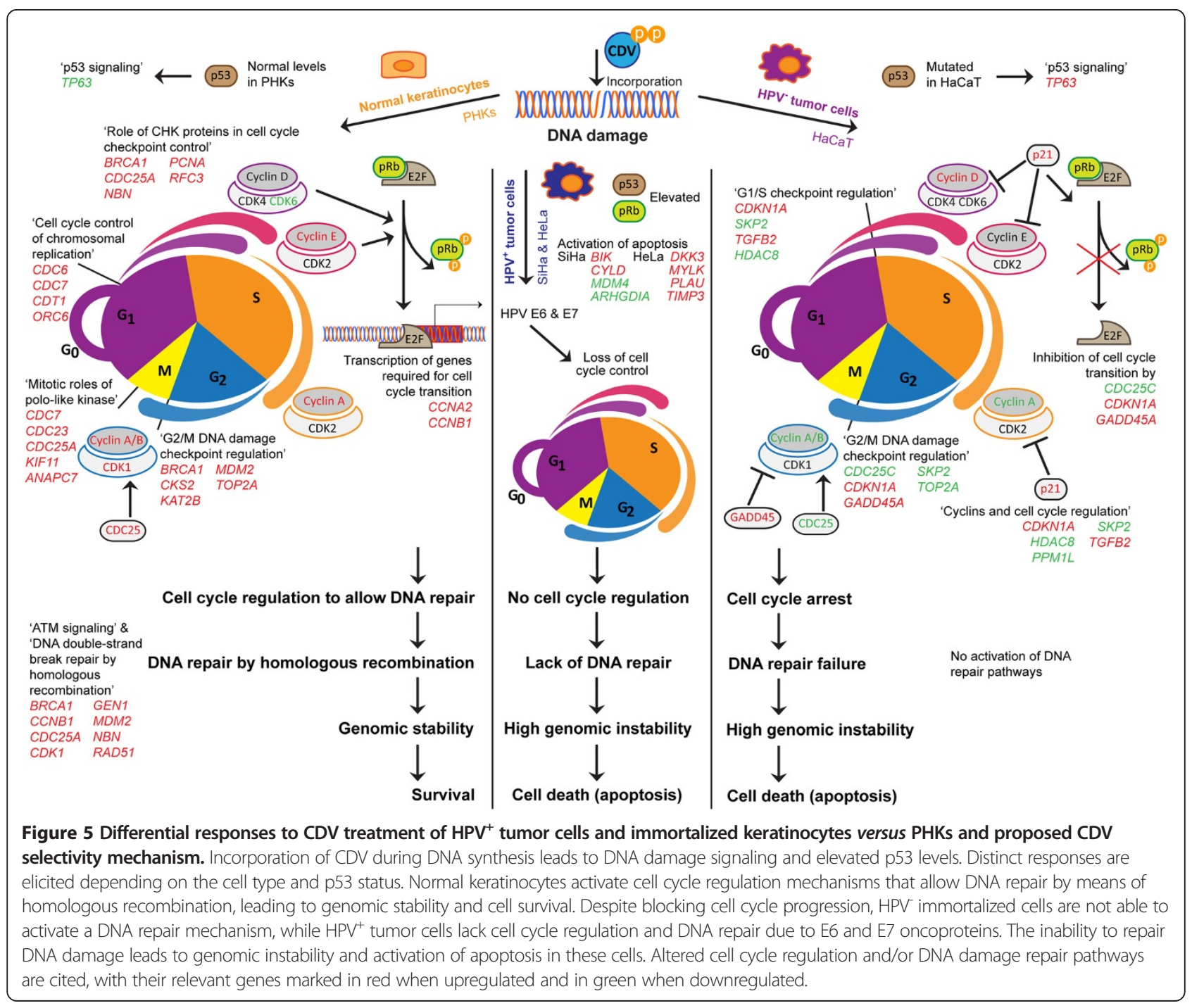


induces accumulation of tumor cells in the S-phase $[19,20]$, and CDVpp, an analogue of deoxycytidinetriphosphate, can be incorporated into cellular DNA, this drug can cause potentially lethal chromosomal DSBs during DNA replication.

In contrast to normal cells that possess an arsenal of repair pathways and cell cycle checkpoints to detect and repair DNA damage, cancer cells as well as immortalized keratinocytes have a significantly reduced set of DNA repair pathways for survival, which can be targeted to develop improved treatment strategies [34]. Differences in the response of normal cells and cancer cells to DNA damaging agents also explain the mechanisms by which the nucleoside analogue ganciclovir induces cell death in tumor cells genetically modified to express the herpes simplex virus thymidine kinase gene $[35,36]$.

Here, we identified DE genes linked to cell death and confirmed at the gene expression level apoptosis induction by CDV $[19,21]$. It should be noted that apoptosis induction, accumulation of the cells in the S-phase, increased protein levels of the tumor suppressor proteins p53 and pRb, and decreased cell viability were evidenced following exposure of tumor cells to CDV for 4 to 5 days $[19,20]$, indicating that cells need to accumulate sufficient drug-induced stress before apoptosis takes place. Distinct sets of genes linked to cell death were altered following $72 \mathrm{~h} \mathrm{CDV}$ treatment of $\mathrm{SiHa}$ and HeLa cells, suggesting that although CDV treatment leads to apoptosis in malignant cells, different cells may respond to CDV by modulating distinct sets of genes, most likely reflecting variations in the genetic background between tumor cells. Considering the DE genes involved in cell cycle control and cell death in $\mathrm{HaCaT}$, it can be assumed that apoptosis will be triggered at a later time point than in $\mathrm{HPV}^{+}$cells.

$\mathrm{HPV}^{+}$cells, that are more susceptible to the antiproliferative effects of $\mathrm{CDV}$ than $\mathrm{HPV}^{-}$immortalized keratinocytes and normal keratinocytes [18], divide very rapidly, present a high genomic instability and are defective in cell cycle control and DNA repair mechanisms due to the expression of E6 and E7 oncoproteins. Thus, CDV treatment of cervical cancer cells may result in significant DNA damage during the S-phase that should be responsible for induction of $\mathrm{p} 53$ and apoptosis $[19,20]$.

Some reports claimed that CDV could specifically affect mRNA levels of E6 and E7 [21,37]. Abdulkarim and colleagues found decreased E6 and E7 mRNA levels and reduced protein expression in HPV18 positive cells [21]. However, we were unable to detect E6 protein levels in cervical carcinoma cells, largely due to low endogenous levels of E6, as well as poor quality of available anti-E6 antibodies, in agreement with several reports [38-41]. On the other hand, we did not find a significant alteration in E6 and E7 mRNA levels by quantitative
RT-PCR following treatment with CDV at $50 \mu \mathrm{g} / \mathrm{ml}$ for 1 - to 7 days (Figure 2B). The elevated p53 and $\mathrm{pRb}$ protein levels (reported by $[19,21]$ ) cannot be attributed to increased mRNA expression of these genes according to our microarray and RT-PCR data. It appears that the higher p53 protein levels are the consequence of the DNA damage response following CDV treatment that affects the expression of regulators of p53 (such as MDM2, MDM4, and CDKN1A) resulting in a rapid stabilization of p53 via blocking of its degradation. This is in agreement with previous reports of post-transcriptional regulation of these genes [42], showing a rapid increase in p53 protein concentration without de novo transcription which is particularly advantageous in cells with severely damaged genomes [43].

MDM2 and MDM4 are considered the main cellular antagonist of p53 by limiting its functions [44]. MDM4, that inhibits $\mathrm{p} 53$ by binding its transcriptional activation domain [45], was downregulated in CDV-treated SiHa cells while $M D M 2$ was upregulated in CDVexposed PHKs. Thus, in PHKs, MDM2 is expected to ubiquitinate $\mathrm{p} 53$ and mediate its degradation by nuclear and cytoplasmatic proteasomes. In contrast, in CDVexposed malignant cells, as a consequence of DNA damage accumulation, stabilization of p53 and induction of several pro-apoptotic genes (such as BIK, CYLD, DKK3, $P L A U$, and TIMP3) take place. Activation of BIK through transcriptional pathways (dependent on factors such as E2f and p53 or by epigenetic regulating mechanisms) was described following treatment with anti-cancer drugs [46], and upregulation of $B I K$ is considered as an interventional approach to treat some tumors [47]. The tumor suppressor $C Y L D$ encodes for a deubiquitinase that plays a critical role in the regulation of NF- $\mathrm{kB}$ and activation of caspase-8, its activation being regarded as a therapeutic target in the treatment of cancers [48]. The tumor suppressor DKK3 (RIG) induces apoptosis through mitochondrial pathways in human colon cancer [49] and proapoptotic actions of PLAU in tumor cells have also been described [50]. The tissue inhibitor of metalloproteinases TIMP3 promotes apoptosis involving stabilization of cell death receptors and activation of caspase-8 [51].

Pro-apoptotic activities have been described for GLIPR1 and $M A F B$ that were upregulated in immortalized keratinocytes and $\mathrm{HPV}^{+}$tumor cells. GLIPR1 was shown to induce apoptosis in prostate cancer [52], and to promote MYC ubiquitination and degradation leading to suppression of cancer development [53]. In line with this report, not only upregulation of GLIPR1 but also downregulation of the predicted activities of MYC family members were observed in immortalized cells. Maf proteins were shown to possess tumor suppressor activities through induction of expression of the cell-cycle 
inhibitor p27 [54] and pro-apoptotic activities through inhibition of MYB $[55,56]$ or induction of p53 transcription [57]. MYCN together with MYB were shown to be involved in a reciprocal regulatory loop promoting survival/ proliferation of neuroblastoma cells [58,59]. Both transcription factors are considered potential specific targets for cancer therapy and downregulation of MYCN expression by treatment with antisense or by retinoid acids decreases proliferation of neuroblastoma cells [60]. Several miRNAs, including miR-17-92, are also known to be regulated by MYCN [61], which showed reduced predicted activities in HeLa. MYCN expression was found to be inversely correlated with $D K K 3$ expression [62], which is in line with our HeLa data. Although CDV did not affect MYCN expression, decreased predicted activities of this proto-oncogene support the antiproliferative effects of CDV and apoptosis induction. Activities of MYC members were also reported to be altered by a few conventional cytotoxic drugs that target microtubules, topoisomerases, or DNA, RNA and protein synthesis [63].

Several cyclins and CDKs were differentially modulated by $\mathrm{CDV}$ in $\mathrm{HPV}^{-}$cells (Figure 5). Increased transcription of genes required for cell cycle progression (CCNA2 and CCNB1) suggests that $\mathrm{pRb}$ can be phosphorylated in PHKs leading to release of E2f. Furthermore, cell cycle progression appeared to be blocked in $\mathrm{HaCaT}$ cells as evidenced by upregulation of CDKN1A (p21) that blocks the activity of cyclin-CDK2/4 complexes and GADD45A, whose transcript levels are increased following stressful growth arrest by treatment with DNA-damaging agents. As a consequence of the increased expression of CDKN1A, the complexes cyclinDCDK4/6 and cyclinE-CDK2 are not activated and pRb cannot be phosphorylated in order to release E2f.

Only two genes (AOX1 and CLIC3) were common to all four cell types. Altered expression of CLIC3 following CDV exposure was not associated with any of the functions or pathways modulated by CDV. In contrast, $A O X 1$ was linked to inflammatory response, the only common function found activated in all cell types. However, distinct pathways linked to inflammatory response were affected by CDV in immortalized keratinocytes and $\mathrm{HPV}^{+}$tumor cells versus PHKs. Importantly, 'Acute Phase Response Signaling', a rapid inflammatory response using non-specific defense mechanisms that provides protection not only against microorganisms but also to tissue injury, neoplastic growth or immunological disorders [64], was exclusively identified in $\mathrm{SiHa}, \mathrm{HeLa}$ and $\mathrm{HaCaT}$ cells. Induction of DNA damage by CDV in immortalized cells was associated with acute phase response signaling which is in agreement with data showing that DNA damage leads to an upregulation of immunostimulatory surface ligands and to an increased secretion of pro-inflammatory cytokines in senescent cells [65]. This may result in the activation of acute response signaling in CDV-exposed immortalized cells that may be important in vivo for clearance of the senescent cells. Considering the number of pathways linked to immune response identified in the CDV-treated immortalized cells, it can be inferred that the inflammatory response plays a crucial role in the response of tumor cells to CDV and that activation of the inflammatory response can be regarded as a cellular reaction to CDV-induced stress.

LXRs play a key role in cholesterol transport by inducing the expression of ATP-binding cassette (ABC) transporters involved in cholesterol efflux. These nuclear receptors also control diverse pathways implicated in development, reproduction, metabolism, immunity and inflammation. Recent insights into LXR signaling revealed that targeting activation of the LXR pathway harbor promises for the management of metabolic disorders, chronic inflammatory diseases, cancer, and neurodegenerative diseases [66,67]. Therefore, activation of LXR/ RXR by CDV in immortalized cells might be an important mediator in the inflammatory response induced by CDV in these cells.

Also, Rho GTPase pathways were exclusively identified in immortalized keratinocytes and $\mathrm{HPV}^{+}$tumor cells. Rho GTPase proteins (RhoA-C, Rac1, and Cdc42) function as molecular switches in a variety of signaling pathways following stimulation of cell surface receptors and regulate several biological processes, including cell cycle control, epithelial cell polarity, cell migration, cell survival and angiogenesis [68]. Modulation of Rho GTPase pathways by CDV identified in our microarray data is consistent with a previous report that demonstrated the efficacy of CDV in disrupting invasion of HeLa cells by decreasing CXCR4 expression and inhibiting Rho/ROCK activation [23]. RhoGDP dissociation inhibitors (RhoGDIs) are considered antiapoptotic molecules [69], and different therapeutic strategies that target RhoGDIs have previously been proposed [70]. Thus, modulation of the RhoGDI and Rac signaling pathways by CDV may be important in induction of cell death as evidenced by downregulation of ARHGDIA (one of the RhoGDIs) in SiHa cells.

\section{Conclusion}

In summary, cell cycle checkpoint control and DNA damage repair occur only in PHKs following CDV treatment. $\mathrm{HPV}^{+}$cells are more susceptible to the antiproliferative action of CDV because they are completely unable to respond to $\mathrm{CDV}$-induced stress while $\mathrm{HaCaT}$ cells still can respond via induction of several signaling pathways but they lack proper cell cycle checkpoint and DNA repairing mechanisms. Furthermore, gene expression profiling allowed the identification of several pathways and functions induced or repressed 
following exposure to CDV that were different in PHKs compared to $\mathrm{HPV}^{+}$and/or $\mathrm{HPV}^{-}$cells, including Rho GTPase pathways and 'acute phase response' exclusively activated in immortalized cells. Our data also have implications for the use of CDV in combination with standard therapy for the treatment of cancer cells that rapidly divide and that show a defect in DNA repairing mechanisms. CDV-induced DNA damage will preferentially accumulate in the tumor cells resulting in S-phase arrest and cell death. Moreover, our findings help to explain the selective effect of CDV which has been clearly documented in several case reports and phase II/III clinical studies [11,14-16]. CDV has been used mostly topically to treat HPV-associated diseases showing a selective antiproliferative effect against HPV lesions without being associated with local side-effects on neighboring normal epithelial cells.

The present findings may lay the scientific basis for further studies on functions and pathways found to be differentially affected by CDV in immortalized keratinocytes and $\mathrm{HPV}^{+}$tumor cells versus normal keratinocytes. Furthermore, this detailed microarray analysis generated a source of novel molecular targets for the treatment of HPVassociated diseases and potentially of non-HPV neoplasias. Our data revealed not only interactions between genes that illustrate useful pathways for new therapeutic targets but also for understanding the mechanism of selectivity of CDV. Additional combined genomic and proteomic studies are required to reveal in even more detail the precise mode of action of CDV and related acyclic nucleoside phosphonates as double-acting (antiviral and antiproliferative) drugs.

\section{Additional files}

Additional file 1: Normalization, filtering, and analysis of microarray data derived from CDV treated and untreated cells. Flow chart of the microarray experiments that was applied to obtain our data sets.

Additional file 2: Functional annotations upregulated or downregulated within functional categories following CDV treatment in different cell types. For the functional analysis, categories associated with unrelated cell types and with irrelevant diseases were omitted. The criteria for selection of functional annotations were based on z-score and statistical significance $(P$-value $<0.05)$. The regulation $z$-score predicts whether an identified biological function is activated or inhibited. Positive $z$-scores indicate activation of a biological function, while negative $z$-scores suggest an inhibition. Absolute $z$-score values above 1 are considered significant.

Additional file 3: Canonical pathway analysis. Canonical pathway analysis of changes in gene expression following incubation of SiHa, HeLa, $\mathrm{HaCaT}$, and PHKs with CDV for $72 \mathrm{~h}$. The significance of the associations between the genes from the data sets and the canonical pathways were determined based on two parameters: (i) P-value, calculated by the Fischer's exact test, that determines the probability that there is an association between the genes in the data set and the canonical pathway that cannot be explained by chance alone and (ii) by the ratio of the number of genes from the data set in a given pathway divided by the total number of molecules in the given canonical pathway. P-values $<0.05$ were considered statistically significant. Pathways marked in black in the PHKs data represent the pathways that were exclusively identified in these cells, while pathways marked in grey represent the pathways that were shared among $\mathrm{SiHa}$, HeLa cells and/or HaCaT cells.

Additional file 4: Prediction of transcription factor activities in the different cell types. The activity of the transcription factors was evaluated by P-value and regulation z-score whose calculation is based on relationships with their target genes. The relationships represent experimentally observed gene expression or transcription events associated with a direction of change that result in activation of inhibition (as derived from the literature compiled in the IPKB). The $z$-score predicts the identified transcription factors to be activated (positive $z$-score) or inhibited (negative z-score). Only upstream regulators that showed an absolute $z$-score $>2$ in at least one of the four cell types are represented. P-values $<0.05$ were considered significant.

Additional file 5: Inflammatory response networks. Networks were constructed with IPA software using genes DE and involved in 'inflammatory response' following CDV treatment of (A) SiHa, (B) HeLa, (C) HaCaT, or (D) PHKs.

Additional file 6: Effect of CDV on 'cell cycle' and 'DNA replication,

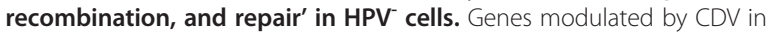
$\mathrm{HaCaT}$ and/or PHKs that are involved in pathways related to 'cell cycle' and 'DNA replication, recombination, and repair'.

\section{Competing interests}

The authors declare they have no competing interests.

\section{Author contributions}

TDS, GA, DT, LN, and RS conceived and designed the experiments. TDS performed the experiments. TDS, GA, DT, LN, and RS analyzed the data. TDS, GA, DT, and RS were responsible for drafting the article. All authors critically revised the article and finally approved the manuscript prior to publication.

\section{Acknowledgements}

We would like to thank Anita Camps and Wim van Dam for their excellent technical assistance and Prof. Jan Balzarini for critical reading of the manuscript. This work was supported by the KU Leuven grants (PF/10/018 and GOA/10/014).

Received: 1 February 2013 Accepted: 17 May 2013

Published: 23 May 2013

\section{References}

1. Bosch FX, de Sanjosé S, Castellsague X, Moreno V, Muñoz N: Epidemiology of human papillomavirus infection and associations with cervical cancer: new opportunities for prevention. In Papillomavirus Research: From Natural History to Vaccines and Beyond. Edited by Campo MS. UK: Caister Academic Press; 2006:19-40.

2. Doorbar J: Molecular biology of human papillomavirus infection and cervical cancer. Clin Sci (Lond) 2006, 110:525-541.

3. zur Hausen $\mathrm{H}$ : Papillomaviruses and cancer: from basic studies to clinical application. Nat Rev Cancer 2002, 2:342-350.

4. Ziegert C, Wentzensen N, Vinokurova S, Kisseljov F, Einenkel J, Hoeckel M, et al: A comprehensive analysis of HPV integration loci in anogenital lesions combining transcript and genome-based amplification techniques. Oncogene 2003, 22:3977-3984.

5. Baker CC, Phelps WC, Lindgren V, Braun MJ, Gonda MA, Howley PM: Structural and transcriptional analysis of human papillomavirus type 16 sequences in cervical carcinoma cell lines. J Virol 1987, 61:962-971.

6. Bodily J, Laimins LA: Persistence of human papillomavirus infection: keys to malignant progression. Trends Microbiol 2011, 19:33-39.

7. Moody CA, Laimins LA: Human papillomavirus oncoproteins: pathways to transformation. Nat Rev Cancer 2010, 10:550-560.

8. Munger K, Basile JR, Duensing S, Eichten A, Gonzalez SL, Grace M, et al: Biological activities and molecular targets of the human papillomavirus E7 oncoprotein. Oncogene 2001, 20:7888-7898.

9. Howie HL, Katzenellenbogen RA, Galloway DA: Papillomavirus E6 proteins. Virology 2009, 384:324-334.

10. De Clercq E, Holy A: Acyclic nucleoside phosphonates: a key class of antiviral drugs. Nat Rev Drug Discov 2005, 4:928-940.

11. Van Pachterbeke C, Bucella D, Rozenberg S, Manigart $Y$, Gilles C, Larsimont D, et al: Topical treatment of CIN 2+ by cidofovir: results of a phase II, 
double-blind, prospective, placebo-controlled study. Gynecol Oncol 2009, 115:69-74.

12. Snoeck R, Noel JC, Muller C, De Clercq E, Bossens M: Cidofovir, a new approach for the treatment of cervix intraepithelial neoplasia grade III (CIN III). J Med Virol 2000, 60:205-209.

13. Koonsaeng S, Verschraegen C, Freedman R, Bossens M, Kudelka A, Kavanagh J, et al: Successful treatment of recurrent vulvar intraepithelial neoplasia resistant to interferon and isotretinoin with cidofovir. J Med Virol 2001, 64:195-198.

14. Stier EA, Goldstone SE, Einstein MH, Jay N, Berry JM, Wilkin T, et al: Safety and efficacy of topical Cidofovir to treat high-grade perianal and vulvar intraepithelial neoplasia in HIV-positive men and women. AIDS 2013, 27:545-551.

15. Tristram A, Fiander A: Clinical responses to Cidofovir applied topically to women with high grade vulval intraepithelial neoplasia. Gynecol Oncol 2005, 99:652-655.

16. Collette DC, Zechel MA: Novel treatment of atypical human papillomavirusassociated epithelial hyperplasia with cidofovir. J Oral Maxillofac Surg 2011, 69:2383-2386.

17. Van Cutsem E, Snoeck R, Van Ranst M, Fiten P, Opdenakker G, Geboes K, et al: Successful treatment of a squamous papilloma of the hypopharynxesophagus by local injections of (S)-1-(3-hydroxy-2phosphonylmethoxypropyl)cytosine. J Med Virol 1995, 45:230-235.

18. Andrei G, Snoeck R, Piette J, Delvenne P, De Clercq E: Antiproliferative effects of acyclic nucleoside phosphonates on human papillomavirus (HPV)-harboring cell lines compared with HPV-negative cell lines. Oncol Res 1998, 10:523-531.

19. Andrei G, Snoeck R, Schols D, De Clercq E: Induction of apoptosis by cidofovir in human papillomavirus (HPV)-positive cells. Oncol Res 2000, 12:397-408

20. Hostetler KY, Rought S, Aldern KA, Trahan J, Beadle JR, Corbeil J: Enhanced antiproliferative effects of alkoxyalkyl esters of cidofovir in human cervical cancer cells in vitro. Mol Cancer Ther 2006, 5:156-159.

21. Abdulkarim B, Sabri S, Deutsch E, Chagraoui H, Maggiorella L, Thierry J, et al: Antiviral agent Cidofovir restores p53 function and enhances the radiosensitivity in HPV-associated cancers. Oncogene 2002, 21:2334-2346.

22. Sirianni N, Wang J, Ferris RL: Antiviral activity of Cidofovir on a naturally human papillomavirus-16 infected squamous cell carcinoma of the head and neck (SCCHN) cell line improves radiation sensitivity. Oral Oncol 2005, 41:423-428.

23. Amine A, Rivera S, Opolon P, Dekkal M, Biard DS, Bouamar H, et al: Novel anti-metastatic action of cidofovir mediated by inhibition of E6/E7, CXCR4 and Rho/ROCK signaling in HPV tumor cells. PLoS One 2009, 4:e5018.

24. Johnson JA, Gangemi JD: Selective inhibition of human papillomavirusinduced cell proliferation by (S)-1-[3-hydroxy-2-(phosphonylmethoxy) propyl]cytosine. Antimicrob Agents Chemother 1999, 43:1198-1205.

25. Oliva J, French SW, Li J, Bardag-Gorce F: Proteasome inhibitor treatment reduced fatty acid, triacylglycerol and cholesterol synthesis. Exp Mol Pathol 2012, 93:26-34.

26. Qin S, Chen J, Tanigawa S, Hou DX: Gene expression profiling and pathway network analysis of hepatic metabolic enzymes targeted by baicalein. J Ethnopharmacol 2012, 140:131-140.

27. Andrei G, van den Oord J, Fiten P, Opdenakker G, De Wolf-Peeters C,

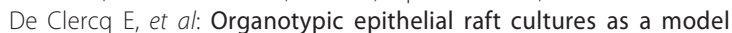
for evaluating compounds against alphaherpesviruses. Antimicrob Agents Chemother 2005, 49:4671-4680.

28. Irizarry RA, Bolstad BM, Collin F, Cope LM, Hobbs B, Speed TP: Summaries of Affymetrix GeneChip probe level data. Nucleic Acids Res 2003, 31:e15.

29. Benjamini $Y$, Hochberg $Y$ : Controlling the false discovery rate - a practical and powerful approach to multiple testing. J Roy Stat Soc B Met 1995, 57:289-300

30. Naesens L, Andrei G, Votruba I, Krecmerova M, Holy A, Neyts J, et al: Intracellular metabolism of the new antiviral compound 1-(S)-[3-hydroxy-2(phosphonomethoxy)propyl]-5-azacytosine. Biochem Pharmacol 2008, 76:997-1005.

31. Dawson Ml, Xia Z: The retinoid X receptors and their ligands. Biochim Biophys Acta 1821, 2012:21-56.

32. DeBerardinis RJ, Lum JJ, Hatzivassiliou G, Thompson CB: The biology of cancer: metabolic reprogramming fuels cell growth and proliferation. Cell Metab 2008, 7:11-20.
33. Chapman JR, Taylor MR, Boulton SJ: Playing the end game: DNA doublestrand break repair pathway choice. Mol Cell 2012, 47:497-510.

34. Aziz K, Nowsheen S, Pantelias G, Iliakis G, Gorgoulis VG, Georgakilas AG: Targeting DNA damage and repair: embracing the pharmacological era for successful cancer therapy. Pharmacol Ther 2012, 133:334-350.

35. Halloran PJ, Fenton RG: Irreversible G2-M arrest and cytoskeletal reorganization induced by cytotoxic nucleoside analogues. Cancer Res 1998, 58:3855-3865.

36. Wei SJ, Chao Y, Hung YM, Lin WC, Yang DM, Shih YL, et al: S- and G2-phase cell cycle arrests and apoptosis induced by ganciclovir in murine melanoma cells transduced with herpes simplex virus thymidine kinase. Exp Cell Res 1998, 241:66-75.

37. Donne AJ, Hampson L, He XT, Rothera MP, Homer JJ, Hampson IN: Cidofovir induces an increase in levels of low-risk and high-risk HPV E6. Head Neck 2009, 31:893-901.

38. Kelley ML, Keiger KE, Lee CJ, Huibregtse JM: The global transcriptional effects of the human papillomavirus E6 protein in cervical carcinoma cell lines are mediated by the E6AP ubiquitin ligase. J Virol 2005, 79:3737-3747

39. Koromilas AE, Li S, Matlashewski G: Control of interferon signaling in human papillomavirus infection. Cytokine Growth Factor Rev 2001, 12:157-170.

40. Masson M, Hindelang C, Sibler AP, Schwalbach G, Trave G, Weiss E: Preferential nuclear localization of the human papillomavirus type 16 E6 oncoprotein in cervical carcinoma cells. J Gen Virol 2003, 84:2099-2104.

41. Zhang HM, Yuan J, Cheung P, Chau D, Wong BW, McManus BM, et al: Gamma interferon-inducible protein 10 induces HeLa cell apoptosis through a p53-dependent pathway initiated by suppression of human papillomavirus type 18 E6 and E7 expression. Mol Cell Biol 2005, 25:6247-6258.

42. Henley SA, Dick FA: The retinoblastoma family of proteins and their regulatory functions in the mammalian cell division cycle. Cell Div 2012, 7:10.

43. Oren M: Regulation of the p53 tumor suppressor protein. J Biol Chem 1999, 274:36031-36034.

44. Moll UM, Petrenko O: The MDM2-p53 interaction. Mol Cancer Res 2003, 1:1001-1008.

45. Perry ME: The regulation of the $\mathrm{p} 53$-mediated stress response by MDM2 and MDM4. Cold Spring Harb Perspect Biol 2010, 2:a000968.

46. Real PJ, Sanz C, Gutierrez O, Pipaon C, Zubiaga AM, Fernandez-Luna JL: Transcriptional activation of the proapoptotic bik gene by E2F proteins in cancer cells. FEBS Lett 2006, 580:5905-5909.

47. Chinnadurai G, Vijayalingam S, Rashmi R: BIK, the founding member of the $\mathrm{BH} 3-$ only family proteins: mechanisms of cell death and role in cancer and pathogenic processes. Oncogene 2008, 27(Suppl 1):S20-S29.

48. Deng LL, Shao YX, Lv HF, Deng HB, Lv FZ: Over-expressing CYLD augments antitumor activity of TRAIL by inhibiting the NF-kappaB survival signaling in lung cancer cells. Neoplasma 2012, 59:18-29.

49. Yang ZR, Dong WG, Lei XF, Liu M, Liu QS: Overexpression of Dickkopf-3 induces apoptosis through mitochondrial pathway in human colon cancer. World J Gastroenterol 2012, 18:1590-1601.

50. Yang H, Choi HJ, Park SH, Kim JS, Moon Y: Macrophage inhibitory cytokine-1 (MIC-1) and subsequent urokinase-type plasminogen activator mediate cell death responses by ribotoxic anisomycin in HCT-116 colon cancer cells. Biochem Pharmacol 2009, 78:1205-1213.

51. Kallio JP, Hopkins-Donaldson S, Baker AH, Kahari VM: TIMP-3 promotes apoptosis in nonadherent small cell lung carcinoma cells lacking functional death receptor pathway. Int J Cancer 2011, 128:991-996.

52. Ren C, Li L, Goltsov AA, Timme TL, Tahir SA, Wang J, et al: mRTVP-1, a novel p53 target gene with proapoptotic activities. Mol Cell Biol 2002, 22:3345-3357.

53. Li L, Ren C, Yang G, Fattah EA, Goltsov AA, Kim SM, et al: GLIPR1 suppresses prostate cancer development through targeted oncoprotein destruction. Cancer Res 2011, 71:7694-7704.

54. Reza HM, Nishi H, Kataoka K, Takahashi Y, Yasuda K: L-Maf regulates p27kip1 expression during chick lens fiber differentiation. Differentiation 2007, 75:737-744.

55. Hegde SP, Zhao J, Ashmun RA, Shapiro LH: c-Maf induces monocytic differentiation and apoptosis in bipotent myeloid progenitors. Blood 1999, 94:1578-1589.

56. Peng S, Lalani S, Leavenworth JW, Ho IC, Pauza ME: c-Maf interacts with c-Myb to down-regulate $\mathrm{BCl}-2$ expression and increase apoptosis in peripheral CD4 cells. Eur J Immunol 2007, 37:2868-2880. 
57. Hale TK, Myers C, Maitra R, Kolzau T, Nishizawa M, Braithwaite AW: Maf transcriptionally activates the mouse p53 promoter and causes a p53dependent cell death. J Biol Chem 2000, 275:17991-17999.

58. Gualdrini F, Corvetta D, Cantilena S, Chayka O, Tanno B, Raschella G, et al: Addiction of MYCN amplified tumours to B-MYB underscores a reciprocal regulatory loop. Oncotarget 2010, 1:278-288.

59. Sottile F, Gnemmi I, Cantilena S, D'Acunto WC, Sala A: A chemical screen identifies the chemotherapeutic drug topotecan as a specific inhibitor of the B-MYB/MYCN axis in neuroblastoma. Oncotarget 2012, 3:535-545.

60. Lu X, Pearson A, Lunec J: The MYCN oncoprotein as a drug development target. Cancer Lett 2003, 197:125-130.

61. Bonauer A, Dimmeler S: The microRNA-17-92 cluster: still a miRacle? Cell Cycle 2009, 8:3866-3873.

62. De Brouwer S, Mestdagh P, Lambertz I, Pattyn F, De PA, Westermann F, et al: Dickkopf-3 is regulated by the MYCN-induced miR-17-92 cluster in neuroblastoma. Int J Cancer 2012, 130:2591-2598.

63. Frenzel A, Zirath H, Vita M, Albihn A, Henriksson MA: Identification of cytotoxic drugs that selectively target tumor cells with MYC overexpression. PLoS One 2011, 6:e27988.

64. Gabay C, Kushner I: Acute-phase proteins and other systemic responses to inflammation. N Engl J Med 1999, 340:448-454.

65. Sperka T, Wang J, Rudolph KL: DNA damage checkpoints in stem cells, ageing and cancer. Nat Rev Mol Cell Biol 2012, 13:579-590.

66. Jakobsson T, Treuter E, Gustafsson JA, Steffensen KR: Liver X receptor biology and pharmacology: new pathways, challenges and opportunities. Trends Pharmacol Sci 2012, 33:394-404.

67. El Roz A, Bard JM, Huvelin JM, Nazih H: LXR agonists and ABCG1-dependent cholesterol efflux in MCF-7 breast cancer cells: relation to proliferation and apoptosis. Anticancer Res 2012, 32:3007-3013.

68. Rathinam R, Berrier A, Alahari SK: Role of Rho GTPases and their regulators in cancer progression. Front Biosci 2011, 16:2561-2571.

69. Zhang B, Zhang Y, Dagher MC, Shacter E: Rho GDP dissociation inhibitor protects cancer cells against drug-induced apoptosis. Cancer Res 2005, 65:6054-6062

70. Lazer G, Katzav S: Guanine nucleotide exchange factors for RhoGTPases: good therapeutic targets for cancer therapy? Cell Signal 2011, 23:969-979.

doi:10.1186/1755-8794-6-18

Cite this article as: De Schutter et al: Cidofovir selectivity is based on the different response of normal and cancer cells to DNA damage. BMC Medical Genomics 2013 6:18.

\section{Submit your next manuscript to BioMed Central and take full advantage of:}

- Convenient online submission

- Thorough peer review

- No space constraints or color figure charges

- Immediate publication on acceptance

- Inclusion in PubMed, CAS, Scopus and Google Scholar

- Research which is freely available for redistribution 